\title{
Final Report \\ US Department of Energy \\ DE-FG02-99ER45797
}

This is the Final Report for BES grant DE-FG02-99ER45797 covering the period from 15 September 2006 through 14 September 2009.

This grant focused on atomistic studies of the dynamics of grain boundaries in metals performed largely using molecular dynamics simulations. In the following, we summarize the major scientific output of these studies.

1) Grain growth and other types of capillarity-driven grain boundary migration depends on both the grain boundary thermodynamics as well as on the intrinsic mobility of the boundaries. While the latter was the focus of the DOE grant during the previous period, one of the foci of this period was on the appropriate thermodynamics - in particular the grain boundary stiffness. The grain boundary stiffness is the sum of the grain boundary free energy and its second variation with respect to grain boundary inclination. In our first paper on this topic (Hao Zhang, Danxu Du, David J. Srolovitz, and Mikhail I. Mendelev, Determination of grain boundary stiffness from molecular dynamics simulation, Applied Physics Letters 88, 121927 2006) we reported on the quantitative prediction of grain boundary stiffness as a function of boundary inclination using molecular dynamics simulations in a series of $\Sigma 5$ [001] tilt grain boundaries. We showed that the grain boundary stiffness exhibits a large anisotropy -- of the same order of magnitude as that of the grain boundary mobility. Surprisingly, the product of the grain boundary stiffness and grain boundary mobility was found to be nearly isotropic. This quantity, known as the reduced grain boundary mobility is the proportionality constant between the grain boundary velocity and the mean curvature of the grain boundary in the grain boundary equation of motion. We wrote a second paper on this topic that focused on a new approach for determining the interface stiffness (Hao Zhang and David J. Srolovitz, Properties and determination of the interface stiffness, Acta Materialia 55 467-471, 2007). In crystalline materials, the grain boundary stiffness is a tensor. We examined several basic issues related to the properties of the grain boundary stiffness, especially the determination of the grain boundary stiffness in particular directions (i.e. the commonly used scalar form of the grain boundary stiffness). We demonstrated that of the five parameters that describe an arbitrary grain boundary, only those describing the inclination are crucial for the scalar stiffness. We also examined the influence of crystal symmetry on the stiffness tensor for both free surfaces and grain boundaries and showed that this results in substantial simplifications for cases in which interfaces possess mirror or rotational symmetries. Finally, we developed an efficient method for determining the full grain boundary stiffness tensor using atomistic simulations.

2) As described above, one of the reasons for focusing on grain boundary stiffness is the connection between grain boundary thermodynamics and grain 
boundary migration especially in the case of capillarity driven migration or grain growth. We worked with a group of researchers at Carnegie Mellon University to make a comparison between grain growth simulations using our grain boundary thermodynamics data and experiments, as reported in K. Barmak, J. Kim, C.-S. Kim, W.E. Archibald, G.S. Rohrer, A.D. Rollett, D. Kinderlehrer, S. Ta'asan, H. Zhang, and D.J. Srolovitz, Grain boundary energy and grain growth in Al films: Comparison of experiments and simulations, Scripta Materialia 54, 1059-1063 2007. In this work, we compared the relative free energies of [111] tilt boundaries in 1.7- $\mu \mathrm{m}$-thick Al films with boundary enthalpies obtained via molecular dynamics simulations. Grain growth studies in 25 and 100-nm-thick Al films were also compared with simulations. This work focused on a critical assessment of the agreement and differences between the experiments and simulations.

3) A separate focus of the supported research looked at shear-driven grain boundary migration - a topic that became very "hot" around that time because of some interesting findings by Cahn and Mishin. We note that this work complemented earlier work under BES funding that used normal stresses or curvature to drive migration. The results of this work were published in Philosophical Magazine (H. Zhang, D. Du and D. J. Srolovitz, Effects of boundary inclination and boundary type on shear-driven grain boundary migration, Philosophical Magazine 88, 243-256 2008). That study used molecular dynamics simulations to study a bicrystal to which a fixed shear rate was applied parallel to the boundary plane. Under some conditions, grain boundary motion was found to be coupled to the relative tangential motion of the two grains (as per Cahn and Mishin). In order to investigate the generality of this type of coupled shear/boundary motion, simulations were performed for both special (low $\Sigma$ ) and general (non- $\Sigma$ ) [010] tilt boundaries over a wide range of grain boundary inclinations. The data pointed to the existence of two critical stresses: one for coupled shear/boundary motion and the other for grain boundary sliding. For the non- $\Sigma$ boundaries, the critical stress for coupled shear/boundary motion was typically smaller than that for sliding; coupled shear/boundary motion occurred for all inclinations. For $\Sigma 5$ boundaries, for which the critical stress is smaller and depends on boundary inclination, coupled shear/boundary motion occurred for some, but not all inclinations.

4) The major focus of our work during this grant was a detailed consideration of the atomic mechanisms associated with grain boundary migration. Much of this work was performed in collaboration with a group from NIST who had considerable experience in analyzing atom dynamics in liquids - a comparison that we found very insightful. In the first study (Hao Zhang, David J. Srolovitz, Jack F. Douglas, and James A. Warren, Characterization of atomic motion governing grain boundary migration, Physical Review B 74, 115404 2006), we performed molecular dynamics simulations to study atomic motion within stationary and migrating asymmetric tilt grain boundaries. We employed several measures of the "complexity" of the atomic trajectories, including the van Hove 
correlation function, the non-Gaussian parameter, and dynamic entropy - new concepts for grain boundaries but widely used in the study of glass-forming liquids. We found two key types of dynamical events within the grain boundaries (i) string-like cooperative atomic motion parallel to the tilt axis and occurring on a characteristic time scale of $\sim 25$ ps and (ii) atomic motion across the grain boundary plane occurring on a characteristic time scale of $\sim 150 \mathrm{ps}$. The characteristic times associated with each type of event decreases with increasing driving force for boundary migration. We found evidence for how the driving force biases these types of events, leading to boundary migration. While the string-like atomic motion is an intrinsic feature of grain boundary dynamics and is important for grain boundary migration, it is the second type of event that controls grain boundary migration rates. The next study (Hao Zhang, David J. Srolovitz, Jack F. Douglas, and James A. Warren, Atomic motion during the migration of general [001] tilt grain boundaries in Ni, Acta Materialia 55, 4527-4533 2007), generalized the results of the previous one to consider arbitrary misorientations of [001] tilt boundaries using similar analysis techniques. We demonstrated the generality of the earlier findings of highly cooperative, string-like motion of atoms. However, in this case, the grain boundary migration itself occurred on even longer time scales. Together, these two works show that and how the grain boundary structure and misorientation play a significant role in determining the rate of grain boundary migration. Finally, we focused on the similarities between the nature of the atomic motions in a glass-forming liquid and a grain boundary in Hao Zhang, David J. Srolovitz, Jack F. Douglas, and James A. Warren, Grain boundaries exhibit the dynamics of glass-forming liquids, PNAS 106, 7735-7740 2009. In short, we took seriously the description of polycrystalline materials as composites of crystalline particles or "grains" separated by thin "amorphous" grain boundaries (GBs) - an idea that has been bandied about in the materials community for sometime but not well accepted. Although GBs were exhaustively investigated at low temperatures where the boundaries are relatively ordered and have a well-defined structure, much less was known about these structures at higher temperatures. It is at these temperatures that where boundary migration (boundary mobility) is significant, where the structural tends to be disorder and where standard characterization methods are limited. Fortunately, the time and spatial scales accessible to molecular dynamics simulations were appropriate for investigating the dynamical and structural properties of GBs at elevated temperatures. We exploited MD to explore basic aspects of GB dynamics as a function of temperature. It had long been hypothesized that GBs have features in common with glass-forming liquids. We found remarkable support for this suggestion, as evidenced by the string-like collective atomic motion and transient caging of atomic motion that is common in glass forming liquids. And, as in glass forming liquids grain boundary mobility was found to exhibit non-Arrhenius behavior (as measured in terms of the average rate of large-scale GB displacement). 